\title{
THE STRONG DUAL OF MEASURE ALGEBRAS WITH CERTAIN LOCALLY CONVEX TOPOLOGIES
}

\author{
HOSSEIN JAVANSHIRI and RASOUL NASR-ISFAHANI ${ }^{\bowtie}$
}

(Received 11 January 2012; accepted 2 July 2012; first published online 8 April 2013)

\begin{abstract}
For a locally compact group $\mathcal{G}$, we introduce and study a class of locally convex topologies $\tau$ on the measure algebra $M(\mathcal{G})$ of $\mathcal{G}$. In particular, we show that the strong dual of $(M(\mathcal{G}), \tau)$ can be identified with a closed subspace of the Banach space $M(G)^{*}$; we also investigate some properties of the locally convex space $(M(\mathcal{G}), \tau)$.
\end{abstract}

2010 Mathematics subject classification: primary 43A10; secondary 43A15, 46A03, 46H05.

Keywords and phrases: measure algebra, strict topology, locally compact group, locally convex space.

\section{Introduction and preliminaries}

Throughout this paper, denote by $\mathcal{G}$ a locally compact group with left Haar measure $\lambda_{\mathcal{G}}$ and identity element $e$, and by $M(\mathcal{G})$ the measure algebras of $\mathcal{G}$ defined as in [7]. We denote the topology generated by the total variation norm on $M(\mathcal{G})$ by $n(\mathcal{G})$. For each complex regular Borel measure $\mu$ on $\mathcal{G}$, let $L^{\infty}(|\mu|)$ denote the Banach space of all bounded Borel measurable functions $f$ on $\mathcal{G}$ with the essential supremum norm

$$
\|f\|_{\mu, \infty}=\inf \{\alpha \geq 0:|f| \leq \alpha,|\mu| \text {-almost everywhere }\} .
$$

Consider the product linear space $\prod\left\{L^{\infty}(|\mu|): \mu \in M(\mathcal{G})\right\}$. An element $f=\left(f_{\mu}\right)_{\mu \in M(G)}$ in this product is called a generalised function if $f_{\mu}=f_{v},|\mu|$-a.e. for each $\mu, v \in M(\mathcal{G})$ with $\mu \ll v$; that is, $|\mu|$ is absolutely continuous with respect to $|v|$. It should be noted that this condition implies that

$$
\sup \left\{\left\|f_{\mu}\right\|_{\mu, \infty}: \mu \in M(\mathcal{G})\right\}<\infty
$$

for all generalised functions $f=\left(f_{\mu}\right)_{\mu \in M(\mathcal{G})}$.

Following Wong [21], denote by $\operatorname{GL}(\mathcal{G})$ the $C^{*}$-algebra of all generalised functions endowed with the pointwise operations. Observe that $\mathrm{GL}(\mathcal{G})$ with the involution given by

$$
f^{*}=\left(\overline{f_{\mu}}\right)_{\mu \in M(\mathcal{G})}
$$

The second author's research was supported in part by a grant from IPM (No. 91430417).

(C) 2013 Australian Mathematical Publishing Association Inc. 0004-9727/2013 \$16.00 
and the norm

$$
\|f\|_{\infty}:=\sup \left\{\left\|f_{\mu}\right\|_{\mu, \infty}: \mu \in M(\mathcal{G})\right\}
$$

for $f=\left(f_{\mu}\right)_{\mu \in M(\mathcal{G})} \in \mathrm{GL}(\mathcal{G})$ is a commutative unital $C^{*}$-algebra. Moreover, any bounded continuous function $f$ on $\mathcal{G}$ can be regarded as an element $\left(f_{\mu}\right)_{\mu \in M(\mathcal{G})}$ of $\mathrm{GL}(\mathcal{G})$, where $f_{\mu}:=f$ for all $\mu \in M(\mathcal{G})$.

With an elegant use of the Radon-Nikodym theorem, Wong [21] proved that the duality

$$
\langle\Psi(f), \mu\rangle:=\int_{\mathcal{G}} f_{\mu}(x) d \mu(x) \quad\left(f=\left(f_{\mu}\right)_{\mu \in M(\mathcal{G})} \in \mathrm{GL}(\mathcal{G})\right)
$$

defines a linear isometric mapping from $\operatorname{GL}(\mathcal{G})$ onto $(M(\mathcal{G}), n(\mathcal{G}))^{*}$, the dual space of $M(\mathcal{G})$; see [16] for the same result in the special case where $\mathcal{G}$ is a locally compact abelian group with countable basis. In particular, any $f \in \mathrm{GL}(\mathcal{G})$ can be considered as a bounded linear functional $\Psi(f)$ on $M(\mathcal{G})$.

On the other hand, for some years, considerable interest has been shown by functional and harmonic analysts in the dual problem of double centraliser of a Banach algebra with strict topology. We now present some information about the history of this problem and known results about it. As far as we know the subject, the starting point of this problem is the paper [1] by Buck on the $C^{*}$-algebra $C_{b}(X)$ of bounded continuous functions on a locally compact Hausdorff space $X$ with the strict topology. Recall that $C_{b}(X)$ is the double centraliser algebra $\mathrm{M}\left(C_{0}(X)\right)$ of $C_{0}(X)$, the $C^{*}$-algebra of all functions in $C_{b}(X)$ which vanish at infinity.

Busby [2] introduced a generalised notation for the strict topology $\beta(A)$ on the double centraliser algebra $\mathrm{M}(A)$ of a $C^{*}$-algebra $A$. The topology $\beta(A)$ on $\mathrm{M}(A)$ is generated by the seminorms $\lambda_{a}$ and $\rho_{a}(a \in A)$ defined by

$$
\lambda_{a}(x)=\|a x\| \quad \text { and } \quad \rho_{a}(x)=\|x a\|
$$

for all $x \in \mathrm{M}(A)$. Although Busby investigated some properties of the strict topology in this setting, no mention was made of the strict dual of $\mathrm{M}(A)$. Taylor [18] has proved that the strict dual of $\mathrm{M}(A)$ under the strong topology is a Banach space that is isometrically isomorphic to the Banach space $(A,\|.\|)^{*}$; see [3] for more details and for similar studies in other contexts, see [4, 13, 14, 20].

By Wendel's theorem [19], the measure algebra $M(\mathcal{G})$ is the double centraliser algebra $\mathrm{M}\left(M_{a}(\mathcal{G})\right)$ of $M_{a}(\mathcal{G})$, the closed ideal of $M(\mathcal{G})$ consisting of all absolutely continuous measures with respect to $\lambda_{\mathcal{G}}$. As in the above discussion, we can consider $M(\mathcal{G})$ as a locally convex space with the strict topology. The dual of $M(\mathcal{G})$ with respect to the strict topology $\beta\left(M_{a}(\mathcal{G})\right)$ can be identified with a subspace of $C_{b}(G)$ which is very far away from the space $\operatorname{GL}(\mathcal{G})$ except for the case where $\mathcal{G}$ is finite; see $[3,5]$ for more details.

The purpose of this paper can be summarised as follows. First, we introduce a locally convex topology $\beta(\mathcal{G})$ on $M(\mathcal{G})$; all results relate to $\beta(\mathcal{G})$, but we also introduce a closed subspace $\mathrm{GL}_{0}(\mathcal{G})$ of $\mathrm{GL}(\mathcal{G})$ which can be identified with the strong dual of $M(\mathcal{G})$. As a major result, we show that, except for the trivial case where $\mathcal{G}$ is 
finite, there are infinitely many such locally convex topologies $\tau$ on $M(\mathcal{G})$ for which $\mathrm{GL}_{0}(\mathcal{G})^{*}$ can be considered as the second dual of $(M(\mathcal{G}), \tau)$. We then deduce that $\beta(\mathcal{G})$ is different from the norm topology $n(\mathcal{G})$ on $M(\mathcal{G})$ whenever $\mathcal{G}$ is noncompact. Finally, we investigate some properties of $(M(\mathcal{G}), \beta(\mathcal{G}))$ as a locally convex space.

\section{2. $M(G)$ with a locally convex topology}

We denote by $\mathcal{K}(\mathcal{G})$ the family of all compact subsets in $\mathcal{G}$. For any increasing sequence $\left(K_{n}\right)$ in $\mathcal{K}(\mathcal{G})$ and any increasing sequence $\left(\alpha_{n}\right)$ in $\mathbb{R}^{+}$such that $\alpha_{n} \nearrow \infty$, set

$$
U\left(\left(K_{n}\right),\left(\alpha_{n}\right)\right)=\left\{\mu \in M(\mathcal{G}):|\mu|\left(K_{n}\right) \leq \alpha_{n} \text { for all } n \geq 1\right\},
$$

and note that $U\left(\left(K_{n}\right),\left(\alpha_{n}\right)\right)$ is a convex balanced absorbing set in the space $M(\mathcal{G})$; we denote by $\mathcal{U}(\mathcal{G})$ the family of all sets of the form $U\left(\left(K_{n}\right),\left(\alpha_{n}\right)\right)$. Then it is easy to see that $\mathcal{U}(\mathcal{G})$ is a base of neighbourhoods of zero for a locally convex topology $\beta(\mathcal{G})$ on $M(\mathcal{G})$. Therefore $\beta(\mathcal{G})$ is the topology generated by the family $\left\{p_{U}: U \in \mathcal{U}(\mathcal{G})\right\}$ of seminorms on $M(\mathcal{G})$, where

$$
p_{U}(\mu)=\sup \left\{\alpha_{n}^{-1}|\mu|\left(K_{n}\right): n \geq 1\right\}
$$

for all $\mu \in M(\mathcal{G})$ and $U:=U\left(\left(K_{n}\right),\left(\alpha_{n}\right)\right) \in \mathcal{U}(\mathcal{G})$. In this section, we investigate some properties of the locally convex topology $\beta(\mathcal{G})$ on $M(\mathcal{G})$. Note that $\beta(\mathcal{G}) \leq n(\mathcal{G})$ and so

$$
(M(\mathcal{G}), \beta(\mathcal{G}))^{*} \subseteq(M(\mathcal{G}), n(\mathcal{G}))^{*} .
$$

We commence this section with the following lemma which is a useful tool in subsequent proofs.

Lemma 2.1. Let $\mathcal{G}$ be a locally compact group. Then a subset of $M(\mathcal{G})$ is $n(\mathcal{G})$-bounded if and only if it is $\beta(\mathcal{G})$-bounded.

Proof. The 'only if' part is trivial. To prove the converse, let $B$ be $\beta(\mathcal{G})$-bounded in $M(\mathcal{G})$. Assume towards a contradiction that $B$ is not $n(\mathcal{G})$-bounded. Then there is a sequence $\left(\mu_{n}\right) \subseteq B$ such that $\left\|\mu_{n}\right\|>n$ for all $n \geq 1$. By the regularity of $\mu_{n}$, there is a compact set $B_{n}$ in $\mathcal{G}$ such that

$$
\left|\mu_{n}\right|\left(B_{n}\right) \geq n
$$

So, if we set $K_{n}=\bigcup_{i=1}^{n} B_{i}$, then $\left|\mu_{n}\right|\left(K_{n}\right) \geq n$ for all $n \geq 1$ and $\left(K_{n}\right)$ is an increasing sequence in $\mathcal{K}(\mathcal{G})$. Since $B$ is $\beta(\mathcal{G})$-bounded,

$$
B \subseteq \alpha U\left(\left(K_{n}\right),\left(\alpha_{n}\right)\right)
$$

for some $\alpha>0$, where $\alpha_{n}^{2}=n$ for $n \geq 1$. Therefore

$$
n \leq\left|\mu_{n}\right|\left(K_{n}\right)<\alpha \alpha_{n}
$$

for all $n \in N$, a contradiction. 
We denote by $\tau_{b}(\mathcal{G})$ the strong topology on $(M(\mathcal{G}), \beta(\mathcal{G}))^{*}$; that is, the topology of uniform convergence on bounded subsets of $M(\mathcal{G})$ with respect to the weak topology $\sigma\left(M(\mathcal{G}),(M(\mathcal{G}), \beta(\mathcal{G}))^{*}\right)$. We also denote by $\tau_{n}(\mathcal{G})$ the topology induced by the norm topology of the dual Banach space $(M(\mathcal{G}), n(\mathcal{G}))^{*}$ on $(M(\mathcal{G}), \beta(\mathcal{G}))^{*}$; that is, the topology given by the norm defined by

$$
\|L\|=\sup \{|L(\mu)|: \mu \in M(\mathcal{G}),\|\mu\|=1\}
$$

for all $L \in(M(\mathcal{G}), \beta(G))^{*}$. As an immediate consequence of Lemma 2.1 we obtain the following result.

Proposition 2.2. Let $\mathcal{G}$ be a locally compact group. Then the topologies $\tau_{b}(\mathcal{G})$ and $\tau_{n}(\mathcal{G})$ coincide on $(M(\mathcal{G}), \beta(\mathcal{G}))^{*}$.

Proposition 2.3. Let $\mathcal{G}$ be a locally compact group. Suppose that the norm topology coincides with the strict topology. Then $\mathcal{G}$ is compact.

Proof. Consider the $n(\mathcal{G})$-open set $U:=\{\mu \in M(\mathcal{G}):\|\mu\|<1\}$, and note that $U$ is also $\beta(\mathcal{G})$-open. It follows that there is a set $U\left(\left(K_{n}\right),\left(\alpha_{n}\right)\right)$ in $\mathcal{U}(\mathcal{G})$ such that

$$
U\left(\left(K_{n}\right),\left(\alpha_{n}\right)\right) \subseteq U
$$

We show that $\mathcal{G}=K_{m}$ for some $m \geq 1$. To this end, assume towards a contradiction that $\mathcal{G} \backslash K_{m} \neq \emptyset$ for all $m \geq 1$. Choose a natural number $m \geq 1$ with $\alpha_{m}>1$, and a compact subset $K$ of $\mathcal{G} \backslash K_{m}$ such that $\lambda_{\mathcal{G}}(K)>0$. Hence for

$$
v:=\left(\frac{\alpha_{m}}{\lambda_{\mathcal{G}}(K)}\right) \chi_{K} d \lambda_{\mathcal{G}},
$$

we have

$$
|v|\left(K_{n}\right)=0 \text { for all } n<m,
$$

and

$$
|v|\left(K_{n}\right) \leq \alpha_{m} \leq \alpha_{n} \quad \text { for all } n \geq m .
$$

Thus $v \in U\left(\left(K_{n}\right),\left(\alpha_{n}\right)\right)$ whence $v \in U$; that is, $\|v\|<1$. On the other hand, $\alpha_{m}=\|v\|$ which contradicts the fact that $\alpha_{m}>1$.

We now introduce the main object of study of this work.

Definition 2.4. Let $\mathcal{G}$ be a locally compact group. We denote by $\mathrm{GL}_{0}(\mathcal{G})$ the $C^{*}$ subalgebra of $\operatorname{GL}(\mathcal{G})$ consisting of all $f=\left(f_{\mu}\right)_{\mu \in M(\mathcal{G})} \in \mathrm{GL}(\mathcal{G})$ with the property that for each $\varepsilon>0$, there is a compact subset $K$ of $\mathcal{G}$ for which

$$
\left\|f \chi_{\mathcal{G} \backslash K}\right\|_{\infty}<\varepsilon
$$

that is, $\left|f_{\mu}(x)\right|<\varepsilon$ for $\mu$-almost all $x \in \mathcal{G} \backslash K(\mu \in M(\mathcal{G}))$, where $\chi_{\mathcal{G} \backslash K}$ denotes the characteristic function of $\mathcal{G} \backslash K$ on $\mathcal{G}$. 
In what follows, we shall investigate some relations between $\mathrm{GL}_{0}(\mathcal{G})$ and $\beta(\mathcal{G})$. Alongside this work, we have studied various aspects of analysis on $\mathrm{GL}_{0}(\mathcal{G})$ and its dual as well, which we shall publish elsewhere. It should be mentioned that some structures similar to $\mathrm{GL}_{0}(\mathcal{G})$ in the study of Banach algebras related to $\mathcal{G}$ can be seen in several recent works; see, for example, [9, 11, 15].

We denote by $\Psi_{0}$, the restriction of $\Psi$ to $\mathrm{GL}_{0}(\mathcal{G})$. Then $M(\mathcal{G})$ and $\mathrm{GL}_{0}(\mathcal{G})$ are in duality with respect to the natural bilinear map given for each $v \in M(\mathcal{G})$ and $f=\left(f_{\mu}\right)_{\mu \in M(\mathcal{G})} \in \mathrm{GL}(\mathcal{G})$ by

$$
\left\langle\Psi_{0}(f), v\right\rangle=\int_{\mathcal{G}} f_{v} d v,
$$

by what was mentioned in the preliminaries; so, if we denote by $\sigma_{0}(\mathcal{G})$ the weak topology $\sigma\left(M(\mathcal{G}), \mathrm{GL}_{0}(\mathcal{G})\right)$ on $M(\mathcal{G})$ induced by this duality, then $\sigma_{0}(\mathcal{G}) \leq \beta(\mathcal{G}) \leq$ $\tau_{n}(\mathcal{G})$. Moreover, it is clear that $\sigma_{0}(\mathcal{G})=\beta(\mathcal{G})$ if $\mathcal{G}$ is finite. The next result shows that this is an 'if and only if' statement.

Theorem 2.5. Let $\mathcal{G}$ be an infinite locally compact group. Then there exist infinitely many locally convex topologies $\tau$ on $M(\mathcal{G})$ with $\sigma_{0}(\mathcal{G}) \leq \tau \leq \beta(\mathcal{G})$.

Proof. The result will follow from [12] if we show that $\sigma_{0}(\mathcal{G})<\beta(\mathcal{G})$. First, note that the weak topology $\sigma(\mathcal{G}):=\sigma(M(\mathcal{G}), \mathrm{GL}(\mathcal{G}))$ is weaker than $n(\mathcal{G})$ by the infiniteness of $\mathcal{G}$. So, if $\mathcal{G}$ is compact, then $\sigma_{0}(\mathcal{G})$ is weaker than $\beta(\mathcal{G})$; this is because $\sigma_{0}(\mathcal{G})$ coincides with $\sigma(\mathcal{G})$, and by Proposition $2.3, \beta(\mathcal{G})$ coincides with $n(\mathcal{G})$.

Now, suppose that $\mathcal{G}$ is noncompact, and choose a compact subset $B_{1}$ of $\mathcal{G}$ with nonempty interior. Then $\mathcal{G} \backslash B_{1}$ is a nonempty open subset of $\mathcal{G}$, and hence there is a compact subset $B_{2}$ of $\mathcal{G}$ with nonempty interior such that $B_{2} \subseteq \mathcal{G} \backslash B_{1}$. By induction we may find a sequence $\left(B_{n}\right)$ of compact subsets of $\mathcal{G}$ with nonempty interior such that

$$
B_{n+1} \subseteq \mathcal{G} \mid \bigcup_{k=1}^{n} B_{k} \quad \text { for all } n \geq 1 \text {. }
$$

Now set

$$
K_{n}=\bigcup_{k=1}^{n} B_{k} \text { for all } n \geq 1 .
$$

Then $\left(K_{n}\right)$ in $\mathcal{K}(\mathcal{G})$ is an increasing sequence and $K_{n+1} \backslash K_{n}$ has nonempty interior for all $n \geq 1$. So, if $\left(\alpha_{n}\right)$ is an increasing sequence of positive real numbers, then $U\left(\left(K_{n}\right),\left(\alpha_{n}\right)\right)$ is a $\beta(G)$-neighbourhood of zero. To complete the proof, we show that $U\left(\left(K_{n}\right),\left(\alpha_{n}\right)\right)$ is not a $\sigma_{0}(\mathcal{G})$-neighbourhood of zero.

For any $n \geq 1$, there exists a probability measure $\mu_{n} \in M(\mathcal{G})$ with

$$
\operatorname{supp}\left(\mu_{n}\right) \subseteq K_{n+1} \backslash K_{n} .
$$

Then the set $\left\{\mu_{n}: n \geq 1\right\}$ is a linearly independent set in $M(\mathcal{G})$. It follows that the subspace

$$
Y:=\left\{\mu \in M(\mathcal{G}): \mu\left(\bigcup_{n=1}^{\infty} K_{n}\right)=0\right\}
$$

is of infinite codimension in $M(\mathcal{G})$. 
Now, if $X$ is a subspace of $M(\mathcal{G})$ contained in the linear span of $U\left(\left(K_{n}\right),\left(\alpha_{n}\right)\right)$, then $X \subseteq Y$, and therefore $X$ has infinite codimension. That is, the linear span $U\left(\left(K_{n}\right),\left(\alpha_{n}\right)\right)$ does not contain any subspace of $M(\mathcal{G})$ with finite codimension. This implies that $U\left(\left(K_{n}\right),\left(\alpha_{n}\right)\right)$ is not a $\sigma_{0}(\mathcal{G})$-neighbourhood of zero.

\section{Dual of $M(G)$ with a locally convex topology}

The next proposition allows us to regard $\mathrm{GL}_{0}(\mathcal{G})$ as a subspace of the dual of $(M(\mathcal{G}), \beta(\mathcal{G}))$.

Proposition 3.1. Let $\mathcal{G}$ be a locally compact group. Then $\Psi_{0}\left(\mathrm{GL}_{0}(\mathcal{G})\right)$ is contained in $(M(\mathcal{G}), \beta(\mathcal{G}))^{*}$.

Proof. Let $g=\left(g_{\mu}\right)_{\mu \in M(\mathcal{G})} \in \mathrm{GL}_{0}(\mathcal{G})$ and $\varepsilon>0$ be given. Choose a sequence $\left(K_{n}\right)$ in $\mathcal{K}(\mathcal{G})$ and $\left(\alpha_{n}\right)$ in $\mathbb{R}^{+}$with $\alpha_{n} \nearrow \infty$ and $\alpha_{1} \geq 2$ such that

$$
\left|g_{\mu}(x)\right| \leq \varepsilon \alpha_{n}^{-2} \quad(n \geq 1),
$$

for $\mu$-almost all $x \in \mathcal{G} \backslash K_{n}(\mu \in M(\mathcal{G}))$. We show that

$$
\left|\left\langle\Psi_{0}(g), \mu\right\rangle\right| \leq \varepsilon \quad \text { for all } \mu \in U\left(\left(K_{n}\right),\left(\alpha_{n}\right)\right)
$$

from which it follows that $\Psi_{0}(g) \in(M(\mathcal{G}), \beta(\mathcal{G}))^{*}$.

To this end, let $\mu \in U\left(\left(K_{n}\right),\left(\alpha_{n}\right)\right)$. Since $g_{\mu}(x)=0$ for $\mu$-almost all $x \in \mathcal{G} \backslash \bigcup_{n=2}^{\infty} K_{n}$, it follows from

$$
\bigcup_{n=2}^{\infty} K_{n}=\bigcup_{n=1}^{\infty}\left(K_{n+1} \backslash K_{n}\right)
$$

that

$$
\begin{aligned}
\left|\int_{\mathcal{G}} g_{\mu}(x) d \mu(x)\right| & \leq \sum_{n=1}^{\infty} \int_{K_{n+1} \backslash K_{n}}\left|g_{\mu}(x)\right| d|\mu|(x) \\
& \leq \sum_{n=1}^{\infty} \varepsilon \alpha_{n}^{-2}|\mu|\left(K_{n+1} \backslash K_{n}\right) .
\end{aligned}
$$

On the other hand,

$$
\begin{aligned}
\sum_{n=1}^{m} \alpha_{n+1}^{-2}|\mu|\left(K_{n+1} \backslash K_{n}\right) & =\sum_{n=1}^{m}\left(\alpha_{n}^{-2}-\alpha_{n+1}^{-2}\right)|\mu|\left(K_{n+1} \backslash K_{1}\right)+\alpha_{m+1}^{-2}|\mu|\left(K_{m+1} \backslash K_{1}\right) \\
& \leq \sum_{n=1}^{m} 2\left(\alpha_{n}^{-1}-\alpha_{n+1}^{-1}\right) \alpha_{n}^{-1}|\mu|\left(K_{n+1}\right)+\alpha_{m+1}^{-2}|\mu|\left(K_{m+1}\right) \\
& <2 \alpha_{1}^{-1}-\alpha_{m+1}^{-1}<1 .
\end{aligned}
$$

Consequently, $\left|\left\langle\Psi_{0}(g), \mu\right\rangle\right| \leq \varepsilon$ as required. 
The main result of this section is the following theorem.

Theorem 3.2. Let $\mathcal{G}$ be a locally compact group. Then the dual of $(M(\mathcal{G}), \beta(\mathcal{G})$ ) endowed with $\tau_{b}(\mathcal{G})$ can be identified with $\mathrm{GL}_{0}(\mathcal{G})$ endowed with the $\|\cdot\|_{\infty}$-topology.

Proof. Let $L$ be a $\beta(G)$-continuous functional on $M(\mathcal{G})$. Then there is a set $U\left(\left(K_{n}\right),\left(\alpha_{n}\right)\right)$ in $\mathcal{U}(\mathcal{G})$ such that

$$
|\langle L, \eta\rangle|<1 \quad \text { for all } \eta \in U\left(\left(K_{n}\right),\left(\alpha_{n}\right)\right) .
$$

Since $\beta(\mathcal{G}) \leq n(\mathcal{G}), L$ is also norm continuous on $M(\mathcal{G})$. Thus, there exists a generalised function $g=\left(g_{\mu}\right)_{\mu \in M(\mathcal{G})} \in \mathrm{GL}(\mathcal{G})$ such that $\Psi(g)=L$ and $\|g\|_{\infty}=\|L\|$. We show that $g \in \mathrm{GL}_{0}(\mathcal{G})$. It suffices to prove that

$$
\left|g_{\mu}(x)\right| \leq \alpha_{n}^{-1}
$$

for all $n \geq 1$ and $\mu$-almost everywhere $x \in \mathcal{G} \backslash K_{n}(\mu \in M(\mathcal{G}))$.

To this end, assume towards a contradiction that there exist $m \geq 1$ and $\mu \in M(\mathcal{G})$ such that $|\mu|(B)>0$, where

$$
B=\left\{x \in \mathcal{G} \backslash K_{m}:\left|g_{\mu}(x)\right|>\alpha_{m}^{-1}\right\} .
$$

Set $v:=|\mu|(B)^{-1}|\mu| \chi_{B}$. Then $v$ is a probability measure in $M(\mathcal{G})$ with $\operatorname{supp}(v) \subseteq B$. Now, let $h_{v}$ be a $v$-measurable function on $\mathcal{G}$ such that $\left|g_{v}\right|=h_{\nu} g_{v}$. Then

$$
\begin{aligned}
\alpha_{m} \alpha_{m}^{-1} & \leq \int_{B} \alpha_{m}\left|g_{\mu}\right| d v=\int_{B} \alpha_{m}\left|g_{v}\right| d v \\
& =\left|\int_{\mathcal{G}} g_{v} d \zeta\right|=\left|\int_{\mathcal{G}} g_{\zeta} d \zeta\right| \\
& =|\langle g, \zeta\rangle|=|\langle L, \zeta\rangle|,
\end{aligned}
$$

where $\zeta:=\alpha_{m} h_{v} v$. But

$$
|\zeta|\left(K_{n}\right) \leq \alpha_{m} \leq \alpha_{n} \text { for all } n \geq m
$$

and

$$
|\zeta|\left(K_{n}\right) \leq|\zeta|\left(K_{m}\right)=0 \quad \text { for all } n<m .
$$

Thus $\zeta \in U\left(\left(K_{n}\right),\left(\alpha_{n}\right)\right)$ whence

$$
1=\alpha_{m} \alpha_{m}^{-1} \leq\{\langle L, \zeta\rangle\}<1,
$$

a contradiction. This implies that

$$
\Psi_{0}\left(\mathrm{GL}_{0}(\mathcal{G})\right)=(M(\mathcal{G}), \beta(\mathcal{G}))^{*} .
$$

Moreover, $\|g\|_{\infty}=\|L\|$ for all $g \in \mathrm{GL}_{0}(\mathcal{G})$. Now, invoke Proposition 2.2 to complete the proof. 
Let us remark that $\sigma_{0}(\mathcal{G}) \leq \beta(\mathcal{G}) \leq n(\mathcal{G})$ and therefore

$$
\Psi_{0}\left(\mathrm{GL}_{0}(\mathcal{G})\right)=\left(M(\mathcal{G}), \sigma_{0}(\mathcal{G})\right)^{*} \subseteq(M(\mathcal{G}), \beta(\mathcal{G}))^{*} \subseteq(M(\mathcal{G}), n(\mathcal{G}))^{*} .
$$

CoRollary 3.3. Let $\mathcal{G}$ be a locally compact group and $\tau$ be a locally convex topology on $M(\mathcal{G})$ with $\sigma_{0}(\mathcal{G}) \leq \tau \leq \beta(\mathcal{G})$. Then the dual of $(M(\mathcal{G}), \tau)$ endowed with the strong topology can be identified with $\mathrm{GL}_{0}(\mathcal{G})$ endowed with the $\|\cdot\|_{\infty}$-topology.

For any $\mu \in M(\mathcal{G})$, let $L^{\infty}(|\mu|)=L^{1}(|\mu|)^{*}$ be endowed with the weak* topology. The product of the weak* topologies on $\prod\left\{L^{\infty}(|\mu|): \mu \in M(\mathcal{G})\right\}$ is called the weak* operator topology on $\mathrm{GL}(\mathcal{G})$ and we denote by $\tau_{w^{*}}(\mathcal{G})$ the relative weak ${ }^{*}$ operator topology on $\mathrm{GL}_{0}(\mathcal{G})$. Also, let $\sigma_{0}^{*}(\mathcal{G})$ be the weak topology on $(M(\mathcal{G}), \beta(\mathcal{G}))^{*}$. Here, we investigate another trait of the map $f \mapsto \Psi_{0}(f)$ from $\mathrm{GL}_{0}(\mathcal{G})$ onto $(M(\mathcal{G}), \beta(\mathcal{G}))^{*}$. First, let us recall that for each $\mu \in M(\mathcal{G})$,

$$
L^{1}(\mu)=\{v \in M(\mathcal{G}): v \ll \mu\}
$$

by the Radon-Nikodym theorem.

THeorem 3.4. Let $\mathcal{G}$ be a locally compact group. The map $f \mapsto \Psi_{0}(f)$ is a homeomorphism from $\mathrm{GL}_{0}(\mathcal{G})$ with the topology $\tau_{w^{*}}(\mathcal{G})$ onto $(M(\mathcal{G}), \beta(\mathcal{G}))^{*}$ with the topology $\sigma_{0}^{*}(\mathcal{G})$.

Proof. Suppose $\left(f^{\alpha}\right)$ is a net in $\mathrm{GL}_{0}(\mathcal{G})$ such that $f^{\alpha} \rightarrow f \in \mathrm{GL}_{0}(\mathcal{G})$ in the topology $\tau_{w^{*}}(\mathcal{G})$. Then $f_{\mu}^{\alpha} \rightarrow f_{\mu}$ in the weak* topology of $L^{\infty}(|\mu|)$ for all $\mu \in M(\mathcal{G})$. In particular,

$$
\int_{\mathcal{G}} f_{\mu}^{\alpha} d \mu \rightarrow \int_{\mathcal{G}} f_{\mu} d \mu
$$

Hence $\left\langle\Psi_{0}\left(f^{\alpha}\right), \mu\right\rangle \rightarrow\left\langle\Psi_{0}(f), \mu\right\rangle$ for all $\mu \in M(\mathcal{G})$; so

$$
\Psi_{0}\left(f^{\alpha}\right) \rightarrow \Psi_{0}(f)
$$

in the $\sigma_{0}^{*}(\mathcal{G})$ topology of $(M(\mathcal{G}), \beta(\mathcal{G}))^{*}$.

On the other hand, if

$$
\Psi_{0}\left(f^{\alpha}\right) \rightarrow \Psi_{0}(f)
$$

in the topology $\sigma_{0}^{*}(\mathcal{G})$ of $(M(\mathcal{G}), \beta(\mathcal{G}))^{*}$, and $v \in M(\mathcal{G})$ with $v \ll \mu$, then

$$
\begin{aligned}
\int_{\mathcal{G}} f_{\mu}^{\alpha} d v & =\int_{\mathcal{G}} f_{v}^{\alpha} d v=\left\langle\Psi_{0}\left(f^{\alpha}\right), v\right\rangle \\
& \rightarrow\left\langle\Psi_{0}(f), v\right\rangle=\int_{\mathcal{G}} f_{v} d v \\
& =\int_{\mathcal{G}} f_{\mu} d v .
\end{aligned}
$$

That is, $f_{\mu}^{\alpha} \rightarrow f_{\mu}$ in the weak ${ }^{*}$ topology of $L^{\infty}(|\mu|)$. 


\section{Some properties of $(M(\mathcal{G}), \beta(\mathcal{G}))$}

We now investigate some properties of the strict topology $\beta(\mathcal{G})$ on $M(\mathcal{G})$. First, let us recall some notions on generalised functions. Let $f$ be a bounded Borel measurable function on $\mathcal{G}$ and $\zeta \in M(\mathcal{G})$. We define the left and right convolutions $l_{\zeta} f$ and $r_{\zeta} f$ by

$$
l_{\zeta} f(x)=\int_{\mathcal{G}} f(y x) d \zeta(y), \quad r_{\zeta} f(x)=\int_{\mathcal{G}} f(x y) d \zeta(y)
$$

for $x \in \mathcal{G}$ whenever these integrals make sense. Note that $\int_{\mathcal{G}} f(y x) d \zeta(y)$ may not be defined for all $x \in \mathcal{G}$; however, by Fubini's theorem, for any $\mu \in M(\mathcal{G})$, it is defined everywhere outside some $|\mu|$-null set. Putting it equal to zero where it is not defined, we obtain a bounded Borel measurable function $l_{\zeta} f$ depending on the $|\mu|$-null set, but it is easy to see that $l_{\zeta} f$ determines uniquely an equivalence class in $L^{\infty}(|\mu|)$. Therefore, we can define $\zeta \circ f \in \prod\left\{L^{\infty}(|\mu|): \mu \in M(\mathcal{G})\right\}$ as

$$
(\zeta \circ f)_{\mu}=l_{\zeta} f_{\zeta * \mu} \quad(\mu \in M(\mathcal{G}))
$$

for all $f=\left(f_{\mu}\right)_{\mu \in M(\mathcal{G})}$ and $\zeta \in M(\mathcal{G})$ such that $\zeta \circ f$ is again a generalised function, and we then have

$$
\langle\zeta \circ f, \mu\rangle=\langle f, \zeta * \mu\rangle
$$

So, $\zeta \circ f$ is the generalised function corresponding to the functional $f \zeta \in M(G)^{*}$. On the other hand, for any $\mu \in M(\mathcal{G})$,

$$
\left\|(\zeta \circ f)_{\mu}\right\|_{\mu, \infty} \leq\|\zeta\|\left\|f_{\zeta * \mu}\right\|_{\zeta * \mu, \infty} .
$$

So, if $f \in \mathrm{GL}_{0}(\mathcal{G})$, then $\zeta \circ f$ is in $\mathrm{GL}_{0}(\mathcal{G})$ and

$$
\|\zeta \circ f\|_{\infty} \leq\|\zeta\|\|f\|_{\infty}
$$

For an extensive study of these notions, see [21, 22].

Proposition 4.1. Let $\mathcal{G}$ be a locally compact group. Then the convolution on $M(\mathcal{G})$ is separately continuous with respect to the weak topology $\sigma_{0}(\mathcal{G})$.

Proof. The $\sigma_{0}(\mathcal{G})$-separate continuity of the convolution follows from the fact that, for any $\zeta \in M(\mathcal{G})$ and $L \in\left(M(\mathcal{G}), \sigma_{0}(\mathcal{G})\right)^{*}$, the linear functional $L \zeta$ on $M(\mathcal{G})$ defined by

$$
\langle L \zeta, \mu\rangle=\langle L, \zeta * \mu\rangle
$$

for all $\mu \in M(\mathcal{G})$ is $\beta(\mathcal{G})$-continuous. So, it is sufficient to prove this phenomenon. To this end, we show that, for any $\zeta \in M(\mathcal{G})$ and $L \in\left(M(\mathcal{G}), \sigma_{0}(\mathcal{G})\right)^{*}$,

$$
\Psi^{-1}(L \zeta)=\zeta \circ \Psi_{0}^{-1}(L)
$$


Indeed, if $\zeta \in M(\mathcal{G})$ and $L \in\left(M(\mathcal{G}), \sigma_{0}(\mathcal{G})\right)^{*}$, then for each $\mu \in M(\mathcal{G})$ and arbitrary Borel subset $A$ of $\mathcal{G}$ we have $\mu_{A}:=\chi_{A} \mu \ll \mu$ and so

$$
\begin{aligned}
\int_{\mathcal{G}} \chi_{A}\left(\Psi^{-1}(L \zeta)\right)_{\mu} d \mu & =\int_{\mathcal{G}}\left(\Psi^{-1}(L \zeta)\right)_{\mu} d \mu_{A} \\
& =\int_{\mathcal{G}}\left(\Psi^{-1}(L \zeta)\right)_{\mu_{A}} d \mu_{A} \\
& =\left\langle L \zeta, \mu_{A}\right\rangle \\
& =\left\langle L, \zeta * \mu_{A}\right\rangle \\
& =\int_{\mathcal{G}}\left(\Psi_{0}^{-1}(L)\right)_{\zeta * \mu_{A}} d \zeta * \mu_{A} \\
& =\int_{\mathcal{G}} l_{\zeta}\left(\Psi_{0}^{-1}(L)\right)_{\zeta * \mu_{A}} d \mu_{A} \\
& =\int_{\mathcal{G}}\left(\zeta \circ \Psi_{0}^{-1}(L)\right)_{\mu_{A}} d \mu_{A} \\
& =\int_{\mathcal{G}} \chi_{A}\left(\zeta \circ \Psi_{0}^{-1}(L)\right)_{\mu} d \mu .
\end{aligned}
$$

Hence $\left(\Psi^{-1}(L \zeta)\right)_{\mu}=\left(\zeta \circ \Psi_{0}^{-1}(L)\right)_{\mu}$ for all $\mu \in M(\mathcal{G})$.

The following proposition presents a necessary and sufficient condition under which the strict topology on $M(\mathcal{G})$ is normable. For this purpose, let us recall some definitions from the theory of locally convex spaces. A locally convex space $(E, \tau)$ is called a barrelled space if any barrel set (that is, a closed convex balanced absorbing set) in $E$ is a neighbourhood of zero; it is called a bornological space when any convex balanced subset that absorbs bounded subsets in $E$ is a neighbourhood of zero.

Proposition 4.2. Let $\mathcal{G}$ be a locally compact group. Suppose that $\tau$ is a locally convex topology on $M(\mathcal{G})$ with $\sigma_{0}(\mathcal{G}) \leq \tau \leq \beta(\mathcal{G})$. Then the following assertions are equivalent:

(a) $(M(\mathcal{G}), \tau)$ is barrelled;

(b) $(M(\mathcal{G}), \tau)$ is bornological;

(c) $(M(\mathcal{G}), \tau)$ is normable;

(d) $n(\mathcal{G})=\beta(\mathcal{G})$;

(e) $\mathcal{G}$ is compact.

Proof. We only need to show that (d) holds if (a) or (b) holds; this follows from Proposition 2.3 together with the fact that any normable space is a bornological space.

Suppose that (a) holds. Since $\sigma_{0}(\mathcal{G}) \leq \tau$, it is easy to see that the unit ball

$$
\mathcal{B}:=\{\mu \in M(\mathcal{G}):\|\mu\| \leq 1\}
$$

is $\tau$-closed. So, $\mathcal{B}$ is a barrel set in $M(\mathcal{G})$, and hence it is a $\tau$-neighbourhood of zero by assumption. That is, $n(\mathcal{G}) \leq \tau$ whence $n(\mathcal{G}) \leq \beta(\mathcal{G})$. Invoke Proposition 2.3 to conclude that $\mathcal{G}$ is compact. 
Now suppose that (b) holds, and let

$$
I:(M(\mathcal{G}), \tau) \rightarrow(M(\mathcal{G}), n(\mathcal{G}))
$$

be the identity. Then $I$ is a bounded map by Corollary 3.3 and the Mackey theorem; see [17, Theorem 13.16]. Since $(M(\mathcal{G}), \tau)$ is a bornological space, $I$ is continuous; see [17, Corollary 21.2]. Thus $n(\mathcal{G})=\beta(\mathcal{G})$, and so another application of Proposition 2.3 shows that $\mathcal{G}$ is compact.

We recall that a locally convex space $(E, \tau)$ is called semireflexive if $(E, \tau)^{* *}=E$.

Proposition 4.3. Let $\mathcal{G}$ be a locally compact group. Suppose that $\tau$ is a locally convex topology on $M(\mathcal{G})$ with $\sigma_{0}(\mathcal{G}) \leq \tau \leq \beta(\mathcal{G})$. Then $(M(\mathcal{G}), \tau)$ is semireflexive if and only if $G$ is discrete.

Proof. Suppose that $\mathcal{G}$ is discrete. Then $M(\mathcal{G})$ can be identified with the space $l^{1}(\mathcal{G})$ of all complex-valued function $\varphi$ on $\mathcal{G}$ such that

$$
\sum_{x \in \mathcal{G}}|\varphi(x)|<\infty .
$$

In view of Corollary 3.3, the dual of $\left(l^{1}(\mathcal{G}), \tau\right)$ equipped with the strong topology can be identified with the Banach space $l_{0}^{\infty}(\mathcal{G})$ equipped with the $\|\cdot\|_{\infty}$-topology, where $l_{0}^{\infty}(\mathcal{G})$ denotes the space of all bounded complex-valued functions $g$ on $\mathcal{G}$ vanishing at infinity. Furthermore, $l_{0}^{\infty}(\mathcal{G})^{*}$ can be identified with $l^{1}(\mathcal{G})$. Thus $\left(l^{1}(\mathcal{G}), \tau\right)^{* *}=l^{1}(\mathcal{G})$.

To prove the converse, let $(M(\mathcal{G}), \tau)$ be semireflexive. Suppose that $B M_{0}(\mathcal{G})$ is the Banach space of all bounded Borel measurable functions on $\mathcal{G}$ that vanish at infinity with the supremum norm. Any $f \in B M_{0}(\mathcal{G})$ can be regarded as an element $\left(f_{\mu}\right)_{\mu \in M(\mathcal{G})}$ of $\mathrm{GL}_{0}(\mathcal{G})$ if we define $f_{\mu}=f$ for $\mu \in M(\mathcal{G})$. But for the space $C_{0}(\mathcal{G})$ of all bounded continuous functions on $\mathcal{G}$ that vanish at infinity,

$$
C_{0}(\mathcal{G})^{\perp}=\left\{m \in \mathrm{GL}_{0}(G)^{*}:\left.m\right|_{C_{0}(\mathcal{G})}=0\right\}=\{0\}
$$

by Corollary 3.3. Thus the Hahn-Banach theorem implies that $C_{0}(\mathcal{G})=\mathrm{GL}_{0}(\mathcal{G})$. Therefore $C_{0}(\mathcal{G})=B M_{0}(\mathcal{G})$ and so $\mathcal{G}$ is discrete.

Corollary 4.4. Let $\mathcal{G}$ be a locally compact group. Suppose that $\tau$ is a locally convex topology on $M(\mathcal{G})$ with $\sigma_{0}(\mathcal{G}) \leq \tau \leq \beta(\mathcal{G})$. Then $(M(\mathcal{G}), \tau)$ is reflexive if and only if $\mathcal{G}$ is finite.

Proof. Since a locally convex space is reflexive if and only if it is barrelled and semireflexive, Propositions 4.2 and 4.3 give the result.

Before we conclude this work with the following result, let us recall that a locally convex space $(E, \tau)$ is said to be a dual space if there exists a locally convex space $\left(E_{0}, \tau_{0}\right)$ such that $(E, \tau)$ coincides with the strong dual of $\left(E_{0}, \tau_{0}\right)$. 
Corollary 4.5. Let $\mathcal{G}$ be a locally compact group. Suppose that $\tau$ is a locally convex topology on $M(\mathcal{G})$ with $\sigma_{0}(\mathcal{G}) \leq \tau \leq \beta(\mathcal{G})$. Then $(M(\mathcal{G}), \tau)$ is a dual space if and only if $\mathcal{G}$ is compact.

Proof. If $\mathcal{G}$ is compact, then $(M(\mathcal{G}), \tau)$ is a barrelled space by Proposition 4.2. This implies that $(M(\mathcal{G}), \tau)$ is a dual space; see [6, Lemma 3.1].

Conversely, suppose that $(M(\mathcal{G}), \tau)$ is a dual space. Since the dual space of $(M(\mathcal{G}), \tau)$ is the normed space $\mathrm{GL}_{0}(\mathcal{G})$, it follows that $(M(\mathcal{G}), \tau)$ is also a normed space; this is because if the dual of a dual space $(E, \tau)$ is normed space, then $(E, \tau)$ is itself a normed space; see [6, Lemma 3.2]. By Proposition 4.2, this can only happen when $\mathcal{G}$ is compact.

\section{Acknowledgements}

The authors would like to express their sincere thanks to the referee for invaluable comments that helped us to improve considerably the presentation of this paper. The second author acknowledges that his research was partially carried out at the IPMIsfahan Branch.

\section{References}

[1] R. C. Buck, 'Bounded continuous functions on a locally compact space', Michigan Math. J. 5 (1958), 95-104.

[2] R. C. Busby, 'Double centralizers and extension of $\mathrm{C}^{*}$-algebras', Trans. Amer. Math. Soc. 132 (1968), 79-99.

[3] J. W. Davenport, 'The strict dual of B*-algebras', Proc. Amer. Math. Soc. 65 (1977), 309-312.

[4] J. Ferrera and A. Prieto, 'The strict topology on spaces of bounded holomorphic functions', Bull. Aust. Math. Soc. 49 (1994), 249-256.

[5] M. Grosser and V. Losert, 'The norm-strict bidual of a Banach algebra and the dual of $C_{u}(\mathcal{G})$ ', Manuscripta Math. 45 (1984), 127-146.

[6] D. Gulick, 'Duality theory for the strict topology', Studia Math. 49 (1974), 195-208.

[7] E. Hewitt and K. Ross, Abstract Harmonic Analysis I (Springer, Berlin, 1970).

[8] N. Isik, J. Pym and A. Ülger, 'The second dual of the group algebra of a compact group', J. Lond. Math. Soc. 35 (1987), 135-148.

[9] A. T. Lau and J. Pym, 'Concerning the second dual of the group algebra of a locally compact group', J. Lond. Math. Soc. 41 (1990), 445-460.

[10] K. B. Laursen and M. Neumann, An Introduction to Local Spectral Theory (Clarendon Press, Oxford, 2000).

[11] M. J. Mehdipour and R. Nasr-Isfahani, 'Compact left multipliers on Banach algebras related to locally compact groups', Bull. Aust. Math. Soc. 79 (2009), 227-238.

[12] J. M. Robertson and H. C. Wiser, 'A note on polar topologies', Canad. Math. Bull. 11 (1968), 607-609.

[13] F. Sentilles and D. Taylor, 'Factorization in Banach algebras and the general strict topology', Trans. Amer. Math. Soc. 142 (1969), 141-152.

[14] J. H. Shapiro, 'The bounded weak star topology and the general strict topology', J. Funct. Anal. 8 (1971), 275-286.

[15] A. I. Singh, ' $L_{0}^{\infty}(\mathcal{G})^{*}$ as the second dual of the group algebra $L^{1}(\mathcal{G})$ with a locally convex topology', Michigan Math. J. 46 (1999), 143-150.

[16] Yu. A. Sreider, 'The structure of maximal ideals in rings of measures with convolution', Math. Sbornik 27 (1950), 297-318. 
[17] C. Swartz, An Introduction to Functional Analysis, Pure and Applied Mathematics, 157 (Marcel Dekker, New York, 1992).

[18] D. C. Taylor, 'The strict topology for double centralizer algebras', Trans. Amer. Math. Soc. 150 (1970), 633-643.

[19] J. G. Wendel, 'Left centralizers and isomorphisms of group algebras', Pacific J. Math. 2 (1952), 251-261.

[20] R. F. Wheeler, 'A survey of Baire measures and strict topologies', Expo. Math. 1 (1983), 97-190.

[21] J. C. Wong, 'Abstract harmonic analysis of generalized functions on locally compact semigroups with applications to invariant means', J. Aust. Math. Soc. 23 (1977), 84-94.

[22] J. C. Wong, 'Convolution and separate continuity', Pacific J. Math 75 (1978), 601-611.

HOSSEIN JAVANSHIRI, Department of Mathematical Sciences, Isfahan University of Technology, Isfahan 84156-83111, Iran

and

Permanent address: Department of Mathematics, Yazd University, PO Box: 89195-741, Yazd-IRAN, Iran

e-mail: h.javanshiri@math.iut.ac.ir

RASOUL NASR-ISFAHANI, Department of Mathematical Sciences, Isfahan University of Technology, Isfahan 84156-83111, Iran

e-mail: isfahani@cc.iut.ac.ir

and

School of Mathematics, Institute for Research in Fundamental Sciences (IPM), PO Box: 19395-5746, Tehran, Iran 